\title{
Maxillary Adenomatoid Odontogenic Tumor: Case Report in a Hospital in Santiago de Chile
}

\author{
Hilda Moris Vidal ${ }^{1 *}$, Nicolás Reyne Silva ${ }^{1}$, Diego Bustamante Correa ${ }^{2}$ and Felipe Astorga Mori ${ }^{3}$ \\ ${ }^{1}$ Hospital Clínico Metropolitano el Carmen Maipú, Santiago, Chile \\ ${ }^{2}$ Oral and Maxillofacial Surgery Resident, Universidad de Chile, Chile \\ ${ }^{3}$ Department of Oral and Maxillofacial Surgery, Hospital Clínico Metropolitano el Carmen Maipú, Santiago, Chile
}

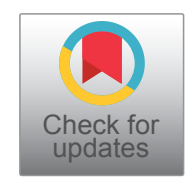

*Corresponding author: Hilda Moris Vial, Hospital Clínico Metropolitano el Carmen, Santiago, Chile

\begin{abstract}
Summary
Objective: Report a case of adenomatoid odontogenic tumor of the maxilla, and to review the literature on the management of this tumor.

Clinical case: 17 -year-old female patient who attended the Maxillofacial Surgery unit at "Hospital del Carmen", Santiago de Chile. Due to increased facial volumen on the right side, persistence of tooth 5.3 and clinical abscence of tooth 1.3. Complementary studies: Orthopantomography and cone beam tomography were performed. The histopathological report of the excisional biopsy showed an adenomatoid odontogenic tumor, associated with tooth 1.3 in the maxillary region.
\end{abstract}

Discussion: The adenomatoid odontogenic tumor is a low prevalence benign tumor that can affect the maxillary and mandibular bone. Treatment should be evaluated based on various factors such as age, location, size, aggressiveness, among others. Conservative surgery either by enucleation or curettage is the treatment of choice, due to its low recurrence and favorable prognosis.

Conclusion: The adenomatoid odontogenic tumor one whose clinical, imaging, histological and location characteristics are very particular, which bring us closer to a definitive diagnosis of this lesion. It is necessary to take a biopsy and a histopathological study to reach the definitive diagnosis of the injury, due to its clinical and imaging characteristics it can be confused with other tumor or odontogenic cyst.

\section{Keywords}

Odontogenic tumors, Adenomatoid odontogenic tumor, Gubernaculum dentis

\section{Introduction}

Odontogenic tumors (OT) are exclusive lesions of the maxillary, mandibular and oral mucosa; derived from mesenchymal and/or ectomesenchymal cells that are or were part of the odontogenesis process [1-5]. They include a spectrum of lesions with diverse biological behavior, ranging from innocuous hamartomatous lesions to aggressive neoplasia [6]. They correspond up to $1 \%$ of all head and neck injuries, the most common being ameloblastomas, myxomas and adenomatoid odontogenic tumor (AOT) [1-5].

AOT corresponds to a benign TO generate by the proliferation of epithelial remains that participate in the odontogenesis process. It is characterized by slow, expansive growth associated with some included teeth, generally maxillary canines [1-3,5]. Unlike the other OT, this lesion is characterized by a mixed radiographic image, with areas of intra-lesional calcification causing large displacement of the teeth and bulging of the tables. Its treatment consists of excision of the lesion and curettage of it. It has a low recurrence rate, if the associated tooth is removed, in addition to curettage or complete enucleation of the lesion $[2,4]$.

The objective of this study is to report a case of AOT in the maxillary region and to review the current literature on the management of this lesion.

\section{Case Description}

17-year-old female, with no diseases or allergies,

Citation: Vidal HM, Silva NR, Correa DB, Mori FA (2021) Maxillary Adenomatoid Odontogenic Tumor: Case Report in a Hospital in Santiago de Chile. Res Rep Oral Maxillofac Surg 5:061. doi. org/10.23937/2643-3907/1710061

Accepted: November 02, 2021; Published: November 04, 2021

Copyright: (C) 2021 Vidal HM, et al. This is an open-access article distributed under the terms of the Creative Commons Attribution License, which permits unrestricted use, distribution, and reproduction in any medium, provided the original author and source are credited. 

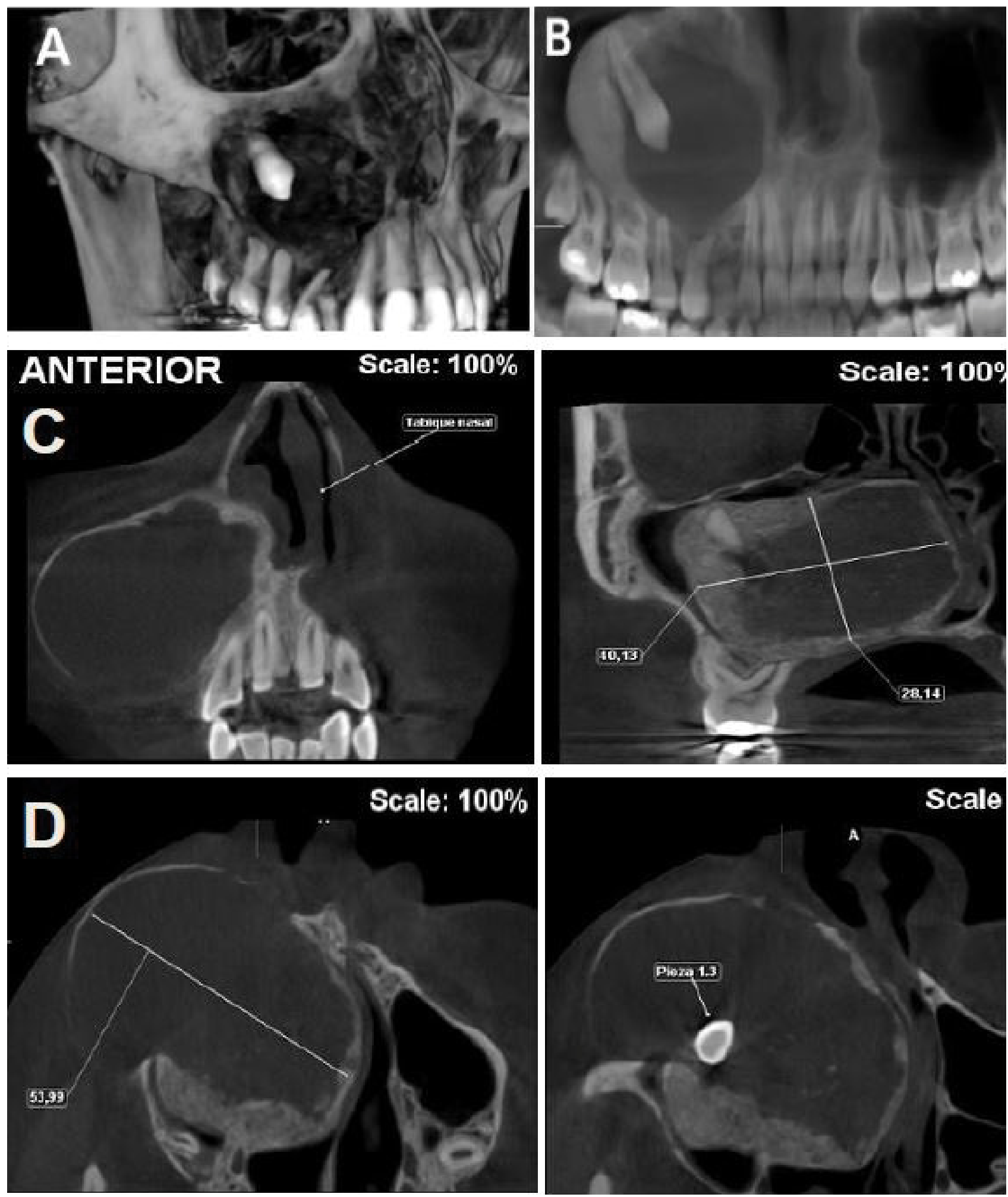

Figure 1: A) 3D reconstruction tumor mass; B) Orthopantomography pre surgical; C) Coronal cut and D) Axial cut.

attends evaluation with the Maxillofacial Surgery team at "Hospital del Carmen" (Santiago, Chile) for progressive right hemimaxillary volume increase. At the extraoral examination, the increase in volume was in the right genian region, causing facial asymmetry, with clear limits and hard on palpation. Intraoral examination revealed persistence of tooth 5.3 and clinical absence of tooth 1.3. Imaging tests were requested: Panoramic radiography and cone beam tomography where a tumor mass was visualized in relation to tooth 1.3 included in the posterosuperior wall of the right maxillary sinus, 5 $\mathrm{cm}$ in diameter, which displaces nasal and sinus mucosa (Figure 1). The condition is explained to the patient, and it is decided to excise the lesion, including an excisional biopsy in the procedure, plus the installation of a drain.

Due to clinical and imaging evaluation, it was decided to perform excisional biopsy. First, an intraoral approach was made at the level of the fundus of the right anterosuperior maxillary vestibule. When accessing 

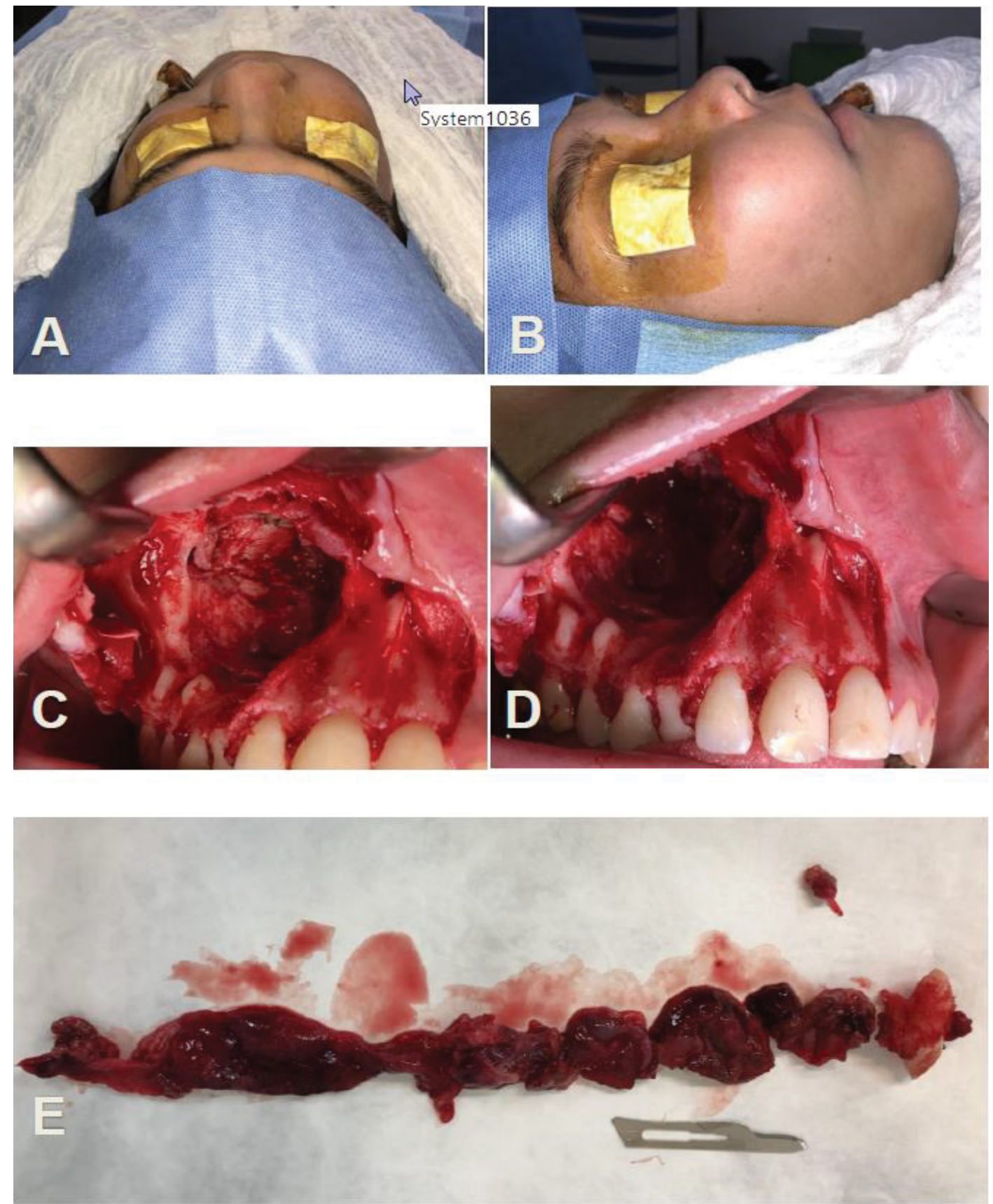

Figure 2: A) Preoperative lateral vision; B) Preoperative superior vision; C) Intraosseous injury; D) Bone cavity post enucleation injury and E) Tooth 1.3 and tumor lesion fragments.

the subperiosteal plane, the lamina was almost nonexistent due to the infectious process of the lesion. By means of osteotomy, a bone window was made to access the lesion and perform its enucleation together with the included canine (Figure 2). Finally, a sample of the excised tissue is taken for a histopathological study, which resulted in a lesion composed of fibrous tissue with epithelial proliferation in islets and areas with glandular duct shapes with multiple calcifications and hyaline content. Which confirmed the diagnosis of AOT. After 2 weeks the patient showed a good clinical and imaging evolution (Figure 3).

\section{Discussion}

Since ever, there has been controversy as to whether
AOT is a cyst or a tumor. AOT is a perfect mimic of the dentigerous cyst (DC), both from a radiological and histopathological point of view, and in this context, numerous case reports of AOT arising from a DC have been reported [7].

AOT is a benign tumor of slow growth, progressive, well defined, with extra and intraoral volume increase, asymptomatic, non-invasive, with a considerable number of lesions that presents perforation of the cortical bone that, frequently, misdiagnosed as a cyst odontogenic. It can displace neighboring teeth and/ or delay their eruption, large lesions, produce root resorption of teeth. It should be added that faster growing lesions show a locally aggressive behavior [1-5]. 

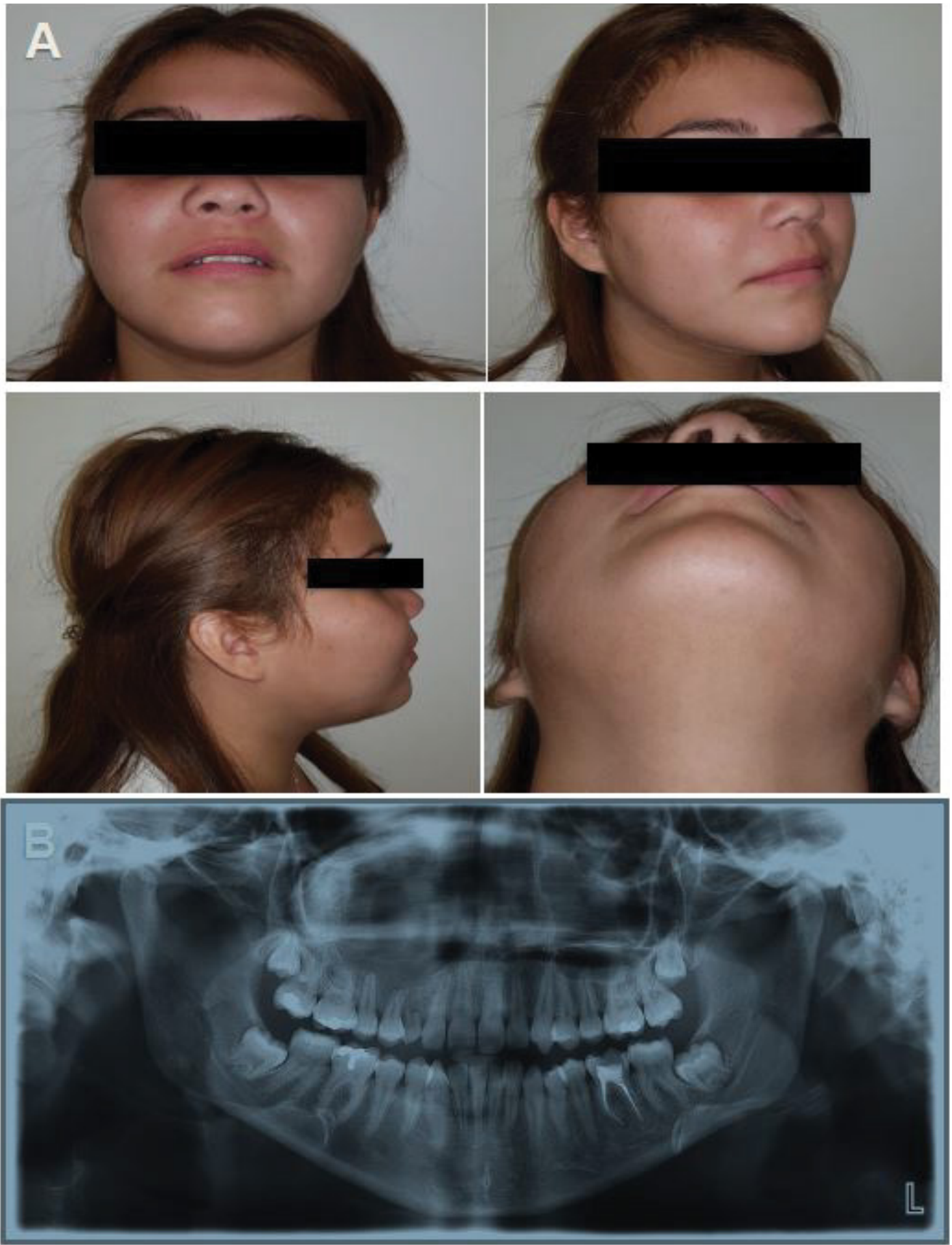

Figure 3: A) Control 2 weeks post operative and B) Orthopantomography post-operative.

It commonly arises in the maxillary bone in a 2:1 ratio with the mandible, with a predilection for the anterior part of the maxilla over the posterior region. The teeth of greatest to least affection are canines, incisors, premolars, molars, being the permanent canine the one that is associated with $51 \%$ of cases, they can also be associated with temporary teeth, supernumerary teeth, non-erupted third molars and even odontomas. $80 \%$ of cases occur in the $2^{\text {nd }}$ and $3^{\text {rd }}$ decade of life approximately, with women under 30 years of age being the most affected, the ratio between women and men of 2:1 [1,2,4,7-9]. In a study from South Africa, AOT accounted for $4 \%$ of all odontogenic tumors, where women were affected six times more often than men [9].
AOT is a rare pathology, with a prevalence close to $3 \%$ of all OT, much less frequent than odontoma, keratocyst and ameloblastoma. It has been suggested that this tumor is more of a hamartoma than a true benign neoplasm, but there is no real evidence to clarify this controversy $[1,2,10]$.

AOT has been classified by the OMS in 2005 as an epithelial odontogenic tumor, with a varied histoarchitectural pattern of a fibrous, mature stroma, without odontogenic ectomesenchyme, with pseudoductal structures and with induction in connective tissue of variable degree $[1,3,5]$.

There are two clinical variants: Intraosseous (97.7\%) 
and extraosseous (2.3\%). The first is subdivided into follicular ( $73 \%$ of cases) and extrafollicular ( $24 \%$ of cases). The Follicular has an intraosseous lesion in relation to a non-erupted tooth, approximately $60 \%$ of cases is a canine, predominantly in the maxilla. And extrafollicular ( $24 \%$ of cases) that has an intraosseous lesion, unrelated to the teeth; and the peripheral or extraosseous variant (0.02-2\% of cases) is rare, it seems to cover a dual pathogenesis, both an "erupted intraosseous" and an "extraosseous" (gingival), where the predilection site is the anteroinferior, there is limited information on its clinical presentation and biological behavior. The most common variant is the follicular type, which is often confused with a DC $[1,2,5,9,11]$.

Due to its slow growth, the intraosseous can expand the bone cortex and cause facial asymmetry such as an increase in volume of hard consistency, without invading soft tissues, not very painful, so the patient consults late when there is already a true deformity $[1,2,5,11]$.

It has been shown that AOT originates from the gubernaculum dentis or also called accessory dental lamina, which is a preformed canal that goes from the dental follicle to the gum and that allows the eruption of the permanent tooth during the mixed dentition period, with effects inductive on connective tissue. It is also suggested that AOT may originate from epithelial debris in close proximity to the crown of a permanent tooth, some tumors may move during tooth eruption along the gubernaculum dentis $[1,2,4,11]$.

Histology of AOT shows greatcellularandarchitectural variability. The most characteristic histopathological study shows cuboidal or spindle-shaped epithelial cells of odontogenic epithelium, which form aggregates or rosette-shaped structures with minimal connective tissue; This structure is surrounded by a well-developed connective tissue stroma with thin-walled vessels, with the presence of a thin epithelial layer and low cuboidal or columnar cells that form structures similar to glandular ducts $[2,4]$.

AOT radiologically is seen as a unilocular cystic lesion, which encloses the non-erupted tooth [12]. The intraosseous variant can show different appearances and, frequently, a radiolucency or a mixed radiolucentradiopaque unilocular image is observed, well demarcated, with a well-defined sclerotic edge of bone, or a smooth cortical bone, associated with the crown and in some cases part of the root of an unexplored tooth imitating a DC, but differs from the latter, since DC does not involve the root [2]. No differences were found when comparing the clinical-radiological characteristics of the follicular, extrafollicular and peripheral variants [3].

Radiographic findings of different tumors show similar-looking lesions, so a differential diagnosis should be made with a DC, calcifying odontogenic cyst, calcifying epithelial odontogenic tumor, unilocular ameloblastoma, ameloblastic fibro-odontoma, keratocystic odontogenic tumor, and an odontoma [2].

Treatment consists of conservative surgical management of the tumor, including extraction of the included tooth and curettage or enucleation, since it is not locally invasive, well encapsulated, gives easy cleavage, and recurrences are rare [2,4].

However, in large tumors, partial or bloc resection may be required, or there may be a risk of pathological fractures. It is convenient to do guided tissue regeneration using lyophilized bone and membranes, especially in large bone cavities [2].

\section{Conclusion}

AOT corresponds to a rare lesion that represents between $2-7 \%$ of OT behind ameloblastoma and keratocyst, its clinical, radiological, histological characteristics and location are very particular, which bring us closer to a definitive diagnosis of this injury, in this clinical case.

As happened with this case, AOT has a higher prevalence in female persons, young people between the 1st and 3rd decade (10-25 years), whose most common location is in the maxilla (2:1) anterosuperior (canine area), appearing generally associated with canine included.

Radiologically, it presents a mixed type of pattern due to intralesional calcifications, well defined associated with canine. However, it does not have a good cleavage plane at the level of the nostril, therefore, drainage is left.

As diagnostic, the histological pattern is found that corresponds to an epithelial proliferation encapsulated with "swirling" cells and forming structure like ducts, with eosinophilic material. It presents variable degrees of inductive changes at the connective tissue level. This tumor can be partially cystic and even in some cases associated with odontomas, for which a good clinical and imaging examination is essential.

Although the characteristics of the lesion are very prone to AOT, it is imperative to take a biopsy and the corresponding histopathological study since in many cases this lesion could be confused with a keratocyst, DC, or an ameloblastoma.

\section{Conflict of Interest}

The authors deny having a conflict of interest in the publication of this work. There are no public or private sources of financing in the realization of this study.

\section{References}

1. Velasco I, Aguilar L, Venables C (2011) Tumor Odontogénico Adenomatoide en Maxilar: Reporte de un Caso y Revisión de la Literatura. Int J Odontostomat 5: 65-69. 
2. Marín Botero ML, Sáenz Rivera E de J, Marín Cardona MN Sánchez Muñoz LB, Castañeda-Peláez DA (2017) Tumor odontogénico adenomatoide. Reporte de un caso y revisión de la literatura. Av Odontoestomatol 33: 161-170.

3. Chrcanovic BR, Gomez RS (2018) Adenomatoid odontogenic tumor: an updated analysis of the cases reported in the literature. Journal of Oral Pathology \& Medicine 48: 10-16.

4. Bravo M, White D, Miles L, Cotton R (2005) Adenomatoid odontogenic tumor mimicking a dentigerous cyst. Int $\mathrm{J}$ Pediatr Otorhinolaryngol 69: 1685-1688.

5. Acharya S, Goyal A, Rattan V, Vaiphei K, Kaur Bhatia S (2014) Dentigerous cyst or adenomatoid odontogenic tumor: clinical radiological and histopathological dilemma. Case Rep Med 2014: 514720.

6. Nel C, Uys A, Robinson L, van Heerden WFP (2021) Multiple adenomatoid odontogenic tumours associated with eight impacted teeth. Oral Radiology 37: 321-327.
7. Srikant N (2010) Cystic adenomatoid odontogenic tumour: The master of disguise. Int J Pediatr Otorhinolaryngol 74: 836-837.

8. Philipsen HP, Samman N, Ormiston IW, Wu PC, Reichart PA (1992) Variants of the adenomatoid odontogenic tumor with a note on tumor origin. J Oral Pathol Med 21: 348-352.

9. Mohamed A, Singh AS, Raubenheimer EJ, Bouckaert MMR (2010) Adenomatoid odontogenic tumour: review of the literature and ananalysis of 33 cases from South Africa. Int J Oral Maxillofac Surg 39: 843-846.

10. Regezi JA, Kerr DA, Courtney RM (1978) Odontogenic tumors: analysis of 706 cases. J Oral Surg 36: 771-778.

11. Philipsen HP, Khongkhunthiang P, Reichart PA (2016) The adenomatoid odontogenic tumour: An update of selected issues. J Oral Pathol Med 45: 394-398.

12. Grover S, Rahim AM, Parakkat NK, Kapoor S, Mittal K, et al. (2015) Cystic Adenomatoid Odontogenic Tumor. Case Rep Dent 2015: 503059. 